\title{
High-pressure structural study of yttrium monochalcogenides from experiment and theory
}

\author{
G. Vaitheeswaran \\ Advanced Centre of Research in High Energy Materials (ACRHEM), University of Hyderabad, Prof. C. R. Rao Road, \\ Gachibowli, Hyderabad 500 046, Andhra Pradesh, India \\ V. Kanchana \\ Department of Physics, Indian Institute of Technology Hyderabad, Ordnance Factory Estate, Yeddumailaram 502205, \\ Andhra Pradesh, India
}

A. Svane and N. E. Christensen

Department of Physics and Astronomy, Aarhus University, DK-8000 Aarhus C, Denmark

J. Staun Olsen

Niels Bohr Institute, Oersted Laboratory, DK-2100 Copenhagen, Denmark

J.-E. Jørgensen

Department of Chemistry, Aarhus University, DK-8000 Aarhus C, Denmark

L. Gerward

Department of Physics, Technical University of Denmark, DK-2800 Lyngby, Denmark

(Received 8 February 2011; published 18 May 2011)

\begin{abstract}
High-pressure powder x-ray diffraction experiments using synchrotron radiation are performed on the yttrium monochalcogenides YS, YSe, and YTe up to a maximum pressure of $23 \mathrm{GPa}$. The ambient $\mathrm{NaCl}$ structure is stable throughout the pressure range covered. The bulk moduli are determined to be 93, 82, and 67 GPa for YS, YSe, and YTe, respectively. First-principles total energy calculations are carried out using the full-potential linear muffin-tin orbital method. The calculated and measured lattice constants and bulk moduli are in good agrement. Under applied pressure, the yttrium monochalcogenides are predicted to undergo a structural transition. Assuming that the high-pressure phase corresponds to the $\mathrm{CsCl}$ crystal structure, transition pressures of 53, 36, and $14 \mathrm{GPa}$ are found for YS, YSe, and YTe, respectively.
\end{abstract}

DOI: 10.1103/PhysRevB.83.184108

PACS number(s): 64.30.Ef, 71.20.Gj, 61.05.cp, 62.20.de

\section{INTRODUCTION}

The yttrium monochalcogenides belong to the large class of binary alloys, which crystallize in the $\mathrm{NaCl}$ (B1) structure at ambient conditions. They are isovalent with the lanthanum monochalcogenides, whose high-pressure properties were recently investigated. ${ }^{1}$ In particular, pressure-induced transformations into the $\mathrm{CsCl}$ (B2) crystal structure were observed for $\mathrm{LaS}, \mathrm{LaSe}$, and LaTe. In the present work, the properties of the $\mathrm{Y} X(X=\mathrm{S}, \mathrm{Se}$, and $\mathrm{Te})$ compounds are investigated under high pressure with the purpose of revealing similarities and differences with the $\mathrm{La} X$ family of compounds, as well as other transition-metal and rare-earth monochalcogenides.

The yttrium monochalcogenides have attracted attention in particular because they are superconductors with transition temperatures around $2 \mathrm{~K},{ }^{2,3}$ which are higher than those of the isostructural lanthanum monochalcogenides. Yttrium sulfide shows a phonon anomaly, ${ }^{4}$ which is suggested to relate to the superconducting nature of this compound. Neutron scattering experiments on YS show that the longitudinal branches exhibit soft-mode regions that closely resemble those found in the structural and electronic analogs $\mathrm{NbC}$ and TiN. ${ }^{4}$ Theoretical works $^{5-8}$ have investigated the electronic structure with emphasis on the clarification of the superconductivity. In particular, the calculated phonon spectra of $\mathrm{YS}^{7,8}$ confirm the observations of strong anomalies in the longitudinal-acoustic branch.
In the present work, high-pressure $\mathrm{x}$-ray diffraction is used to investigate the crystal structure of the yttrium monochalcogenides to pressures up to $23 \mathrm{GPa}$. The measurements are supported by first-principles electronic-structure calculations. To study the possibility of a phase transition under pressure, calculations assuming an alternative $\mathrm{CsCl}$ structure are performed. This choice is motivated by the well-documented occurrence of this phase at high pressures not only for the LaX family ${ }^{1}$ but also for several similar systems, such as the ionic monochalcogenides of $\mathrm{Ca}, \mathrm{Sr}$, and $\mathrm{Ba},{ }^{9-15}$ and the monochalcogenides of several rare earths, such as $\mathrm{Ce}, \mathrm{Pr}, \mathrm{Sm}$, $\mathrm{Eu}$, and $\mathrm{Yb},{ }^{16-22}$ see also theoretical studies. ${ }^{23-25}$

The remainder of the present paper is organized as follows. In Sec. II, details of the experimental and computational procedures are presented. The structural, elastic, and highpressure results are presented in Sec. III. In Sec. IV, the band structures of the yttrium monochalcogenides are discussed. Finally, Sec. V summarizes the results.

\section{EXPERIMENTAL AND COMPUTATIONAL DETAILS}

\section{A. Experimental details}

The YS sample was prepared from stoichiometric amounts of $\mathrm{Y}$ and $\mathrm{Y}_{2} \mathrm{~S}_{3}$. The powder mixture was pressed into pellets and placed in a glassy carbon crucible. The crucible was kept at 


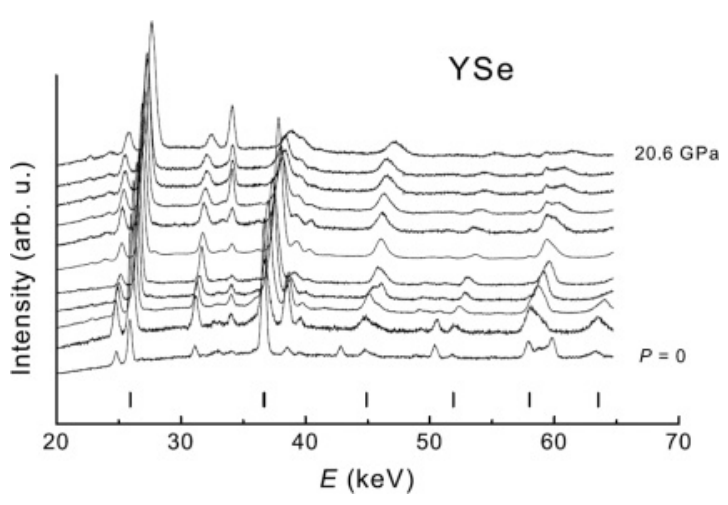

FIG. 1. Energy-dispersive XRD spectra for YSe at selected pressures in the range 0-20.6 GPa. The Bragg angle is $\theta=4.811^{\circ}$. The vertical bars show the peak positions of the B1-type reflections at zero pressure.

about $1700^{\circ} \mathrm{C}$ for $6 \mathrm{~h}$ in $\mathrm{He}$ atmosphere in an induction-heated furnace. The YSe and YTe compounds were prepared directly from the constituent elements: Stoichiometric amounts of Y and $\mathrm{Se}$ or Te powders were mixed and placed in alumina crucibles, which were placed in evacuated quartz ampoules. In the case of YSe, the ampoule was first kept at $850^{\circ} \mathrm{C}$ for $30 \mathrm{~h}$. The sample was then ground in an agate mortar, pressed into a pellet, and again kept at $850{ }^{\circ} \mathrm{C}$ for $40 \mathrm{~h}$. The YTe sample was prepared in the same way as the YSe sample but kept at $650{ }^{\circ} \mathrm{C}$ for $20 \mathrm{~h}$ just once.

High-pressure powder x-ray diffraction (XRD) patterns were recorded using synchrotron radiation and the white-beam method at HASYLAB, Hamburg. The diffractometer, working in energy-dispersive mode, has been described elsewhere. ${ }^{26}$ Pressures up to $23 \mathrm{GPa}$ were obtained in a Syassen-Holzapfeltype diamond-anvil cell. The sample and a small ruby chip were enclosed in a hole of diameter $0.2 \mathrm{~mm}$ in an Inconel gasket. A 16:3: 1 ethanol:methanol:water mixture and in some cases silicone oil were used as pressure-transmitting media. The pressure was determined from the wavelength shift of the ruby line, applying the nonlinear pressure scale of Mao et al. ${ }^{27}$ The uncertainty in the pressure determination is estimated to be $0.1 \mathrm{GPa}$ for pressures below $10 \mathrm{GPa}$. For higher pressures, the uncertainty may be larger because of possible deviations from hydrostatic conditions. The Bragg angle associated with each experimental run was deduced from a zero-pressure spectrum of $\mathrm{NaCl}$.

Figure 1 shows, as an example, a set of energy-dispersive XRD spectra for YSe in the investigated pressure range 0$21 \mathrm{GPa}$. At each pressure, values for the lattice parameter and the unit-cell volume were derived from the peak positions in the XRD spectrum and refined using the PURUM code. ${ }^{28}$ It is seen that the sample is not phase-pure. There are several minor peaks that have been identified as being due to $\mathrm{Y}_{2} \mathrm{Se}_{3}$, which is the stable phase at ambient conditions. This problem is manageable at moderate pressures, since we need just a few diffraction lines to determine the lattice parameter of the cubic phase YSe. However, we have been unable to follow the lattice evolution at pressures above $23 \mathrm{GPa}$ because of the inevitable deterioration of the diffraction spectra at higher pressures, resulting in line broadening and peak overlap. The same argument holds for YS and YTe.
The experimental compression curves have been described by the Birch-Murnaghan equation of state, ${ }^{29}$

$$
P=\frac{3}{2} B_{0}\left(x^{-7 / 3}-x^{-5 / 3}\right)\left[1-\frac{3}{4}\left(4-B_{0}^{\prime}\right)\left(x^{-2 / 3}-1\right)\right],
$$

where $x=V / V_{0}, V$ is the unit-cell volume at pressure $P, V_{0}$ is the unit-cell volume at zero pressure, $B_{0}$ is the zero-pressure bulk modulus, and $B_{0}^{\prime}$ is its pressure derivative. Values of $B_{0}$ and $V_{0}$ were obtained from a nonlinear least-squares fit of Eq. (1) to the experimental data points, while the fixed value $B_{0}^{\prime}=4.0$ was assumed.

\section{B. Theoretical details}

The linear muffin-tin orbital method ${ }^{30}$ in the all electron full-potential (FP-LMTO) implementation of Ref. 31 is employed to calculate the total energies and ground-state properties of the yttrium monochalcogenides. In this method, the crystal is divided into two regions: nonoverlapping muffintin spheres surrounding each atom and the interstitial region between these spheres. An LMTO basis set using two different decay parameters (double $\kappa$ ) was used. The basis set included $(4 s, 5 s, 4 p$, and $4 d)$ orbitals for $\mathrm{Y},(3 s, 3 p$, and $3 d)$ orbitals for $\mathrm{S},(4 s, 4 p, 3 d$, and $4 d)$ orbitals for $\mathrm{Se}$, and $(5 s, 5 p, 4 d$, and $5 d$ ) orbitals for Te, respectively. Inside the muffin-tin spheres, the electron charge density and potentials were expanded in spherical harmonics with an angular momentum cutoff of $l_{\max }=6$. In the interstitial region, plane waves with energies up to 124 Ry (more than 6500 plane waves) were included in the calculation. The ground-state properties were calculated using the generalized gradient approximation $(\mathrm{GGA})^{32}$ for the exchange-correlation energy. Total energies were calculated as a function of volume for a $(20 \times 20 \times 20) k$ mesh in the Brillouin zone.

\section{STRUCTURAL, ELASTIC, AND HIGH-PRESSURE STUDIES}

\section{A. Ground-state and elastic properties}

The measured values of the lattice parameters for $\mathrm{Y} X(X=$ $\mathrm{S}, \mathrm{Se}$, and Te) are given in Table I. The uncertainties, given in parentheses, are the standard deviations of the least-squares fit to the cubic unit cell. It is seen that there is good agreement between the present results and lattice parameters reported previously in the literature. Experimental values of the bulk modulus for $\mathrm{Y} X(X=\mathrm{S}, \mathrm{Se}$, and $\mathrm{Te})$, as determined from the pressure-volume data, are also given in Table I. For the fit to the Birch-Murnaghan relation, it has been assumed that $B_{0}^{\prime}=4.00$ for all compounds. Melcher et al. ${ }^{34}$ have derived the bulk modulus from the measured sound velocity at room temperature and frequency $30 \mathrm{MHz}$ for single crystals of YS. Our experimental value agrees with their value within $4 \%$, which must be considered very satisfactory. To the best of our knowledge, there are no previously published values of the bulk modulus for YSe and YTe.

The calculated equilibrium lattice constant, bulk modulus, and the pressure derivative of the bulk modulus are also included in Table I. The calculated lattice constants are slightly larger than the experimental values, by $\sim 0.2 \%$ for $\mathrm{YS}$ and YTe, and $\sim 0.6 \%$ for YS. Similarly, for the bulk modulus, the agreement between theory and experiment is excellent. The 
TABLE I. Lattice constant (in $\AA$ ), bulk modulus $B_{0}$ (in GPa), and pressure derivative $B_{0}^{\prime}$ of $\mathrm{Y} X(X=\mathrm{S}, \mathrm{Se}, \mathrm{Te})$ as obtained by experiment and theory. The experimental uncertainties are shown in parentheses. The $B_{0}^{\prime}$ value was fixed in the experimental fit.

\begin{tabular}{|c|c|c|c|c|}
\hline Compound & & Lattice constant & $B_{0}$ & $B_{0}^{\prime}$ \\
\hline \multirow[t]{3}{*}{ YS } & Expt., this work & $5.489(7)$ & $93(5)$ & 4.0 \\
\hline & Theory, this work & 5.499 & 101.4 & 4.7 \\
\hline & Other expt. & $5.492,{ }^{\mathrm{a}} 5.495,^{\mathrm{b}} 5.50,^{\mathrm{c}} 5.466^{\mathrm{d}}$ & $96.9^{c}$ & \\
\hline \multirow[t]{3}{*}{ YSe } & Expt., this work & $5.701(5)$ & $82(2)$ & 4.0 \\
\hline & Theory, this work & 5.736 & 85.5 & 4.6 \\
\hline & Other expt. & $5.711,^{\mathrm{a}} 5.703^{\mathrm{e}}$ & & \\
\hline \multirow[t]{3}{*}{ YTe } & Expt., this work & $6.103(8)$ & $67(2)$ & 4.0 \\
\hline & Theory, this work & 6.130 & 67.0 & 4.7 \\
\hline & Other expt. & $6.093,{ }^{\mathrm{a}} 6.095^{\mathrm{f}}$ & & \\
\hline
\end{tabular}

${ }^{\text {a Reference } 3 .}$

${ }^{\mathrm{b}}$ Reference 33 .

${ }^{\mathrm{c}}$ Reference 34 .

${ }^{\mathrm{d}}$ Reference 35 .

${ }^{\mathrm{e}}$ Reference 36.

${ }^{\mathrm{f}}$ Reference 37.

largest deviation is found for YS, where the calculated bulk modulus is $9 \%$ above the measured value. The experimental values of the bulk modulus for $\mathrm{Y} X(X=\mathrm{S}, \mathrm{Se}$, and Te $)$ are consistent, in the sense that there is a perfect scaling of the bulk modulus with the unit-cell volume as illustrated in Fig. 2. This double-logarithmic plot of $V_{0}$ versus $B_{0}$ yields the slope -1.0 , which is typical for ionic compounds. 38,39 The bulk modulus values of the isostructural $\operatorname{La} X, \operatorname{Ce} X$, and $\operatorname{Pr} X(X=\mathrm{S}, \mathrm{Se}$, and $\mathrm{Te})$ compounds are included in Fig. 2.1,18,24,25 They show similar trends to those of the yttrium chalcogenides, i.e., lower bulk modulus for the larger volumes. These similarities corroborate the practice of taking the yttrium chalcogenides as best nonmagnetic reference systems to compare the various physical properties of rare-earth (and actinide) monochalcogenides.

The calculated elastic constants of YS, YSe, and YTe are listed in Table II, where also the experimental elastic constants of YS (Ref. 40) are included. No experimental values are available for YSe and YTe. For comparison, the

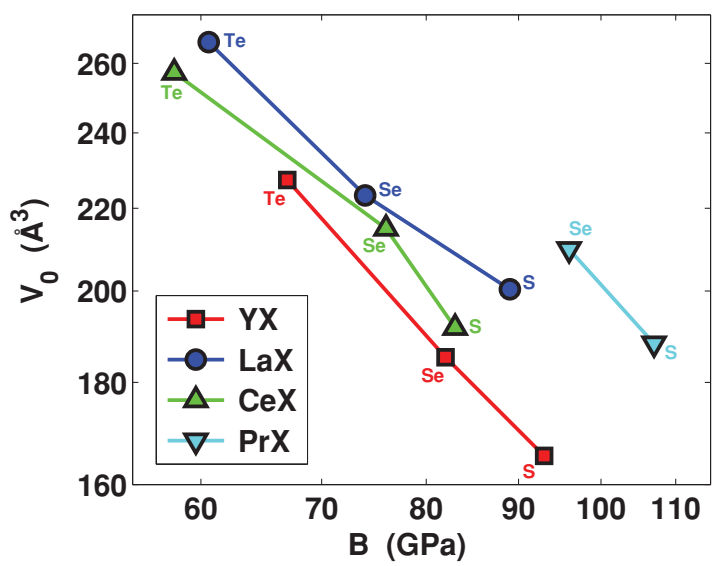

FIG. 2. (Color online) Double logarithmic plot of experimental values of equilibrium volume, $V_{0}$, vs bulk modulus, $B_{0}$, showing the bulk modulus scaling of $\mathrm{Y} X(X=\mathrm{S}, \mathrm{Se}$, and $\mathrm{Te})$ and compared to the $\operatorname{La} X, \operatorname{Ce} X$, and $\operatorname{Pr} X$ homologues. calculated elastic constants of the $\mathrm{La} X$ family of compounds are also quoted. ${ }^{1}$ While the calculated $C_{11}$ elastic constants are somewhat larger in the yttrium compounds than in the lanthanide homologues, the opposite trend is seen for $C_{12}$ and $C_{44}$.

\section{B. High-pressure properties}

The measured pressure-volume relations for YS, YSe, and YTe are shown in Fig. 3 and compared to the theoretical results. The agreement is good overall, however at large pressures the theoretical curves tend to lie increasingly above the experimental data points. For YTe, the calculations furthermore predict a first-order structural transition to occur around $P_{t}=14 \mathrm{GPa}$ (discussed below). No structural phase transformations have been observed in the present experiments for any of the $\mathrm{Y} X$ compounds. However, some of the B1type reflections of YTe disappear at high pressure, which might be a precursor for the transformation. The B1-B2 transformation is observed in the corresponding actinide monochalcogenides,${ }^{26,41}$ where it appears as a rather sluggish transformation. Further experimental work at pressures above

TABLE II. Calculated elastic constants (in GPa) for yttrium monochalcogenides in the B1 structure at the theoretical (GGA) equilibrium volume. For comparison, the experimental results for YS (Ref. 40) are included, as well as the calculated elastic constants of the La homologues (Ref. 1).

\begin{tabular}{llclc}
\hline \hline Compound & $C_{11}$ & $C_{12}$ & \multicolumn{1}{c}{$C_{44}$} & \\
\hline YS & 284.7 & 12.1 & 21.6 & Present \\
& 250 & 20 & 30 & Expt. (Ref. 40) \\
LaS & 227.9 & 18.0 & 22.2 & Theory (Ref. 1) \\
YSe & 239.1 & 9.6 & 11.2 & Present \\
LaSe & 201.6 & 11.4 & 15.7 & Theory (Ref. 1) \\
YTe & 198.9 & 2.0 & 4.2 & Present \\
LaTe & 158.7 & 9.7 & 7.9 & Theory (Ref. 1) \\
\hline \hline
\end{tabular}



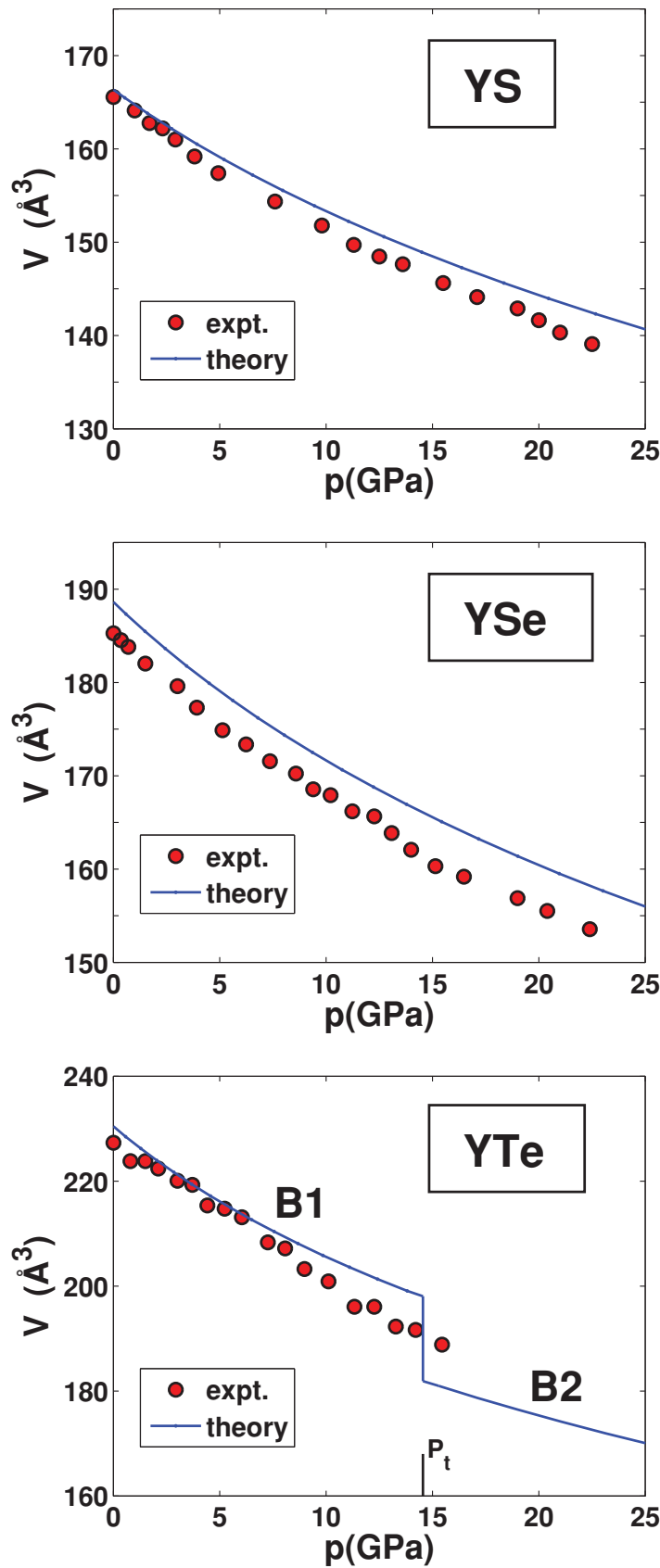

FIG. 3. (Color online) Measured (circles) and calculated (full line) $\mathrm{pV}$ relation of YS, YSe, and YTe. The volumes are the specific volumes for one formula unit, in $\AA^{3}$. For YTe, the predicted structural transition at $P_{t}=14 \mathrm{GPa}$ gives rise to the discontinuous jump in the theoretical curve.

the present maximum of about $15 \mathrm{GPa}$ would be desirable to confirm the B1-B2 transformation predicted by theory. ${ }^{42}$

To study the possible relevance of the $\mathrm{CsCl}$ crystal structure for the yttrium monochalcogenides at high pressure, total energy calculations as a function of volume were performed for the B1 and B2 structures. The results are shown in Fig. 4. From the total energy curves, it emerges that the $\mathrm{NaCl}$ structure is found to provide the lowest overall minimum, thus finding this phase to be stable at ambient pressures, in agreement with the experiments. The calculated structural energy differences between the $\mathrm{NaCl}$ and $\mathrm{CsCl}$ structures are found to be 68, 55, and
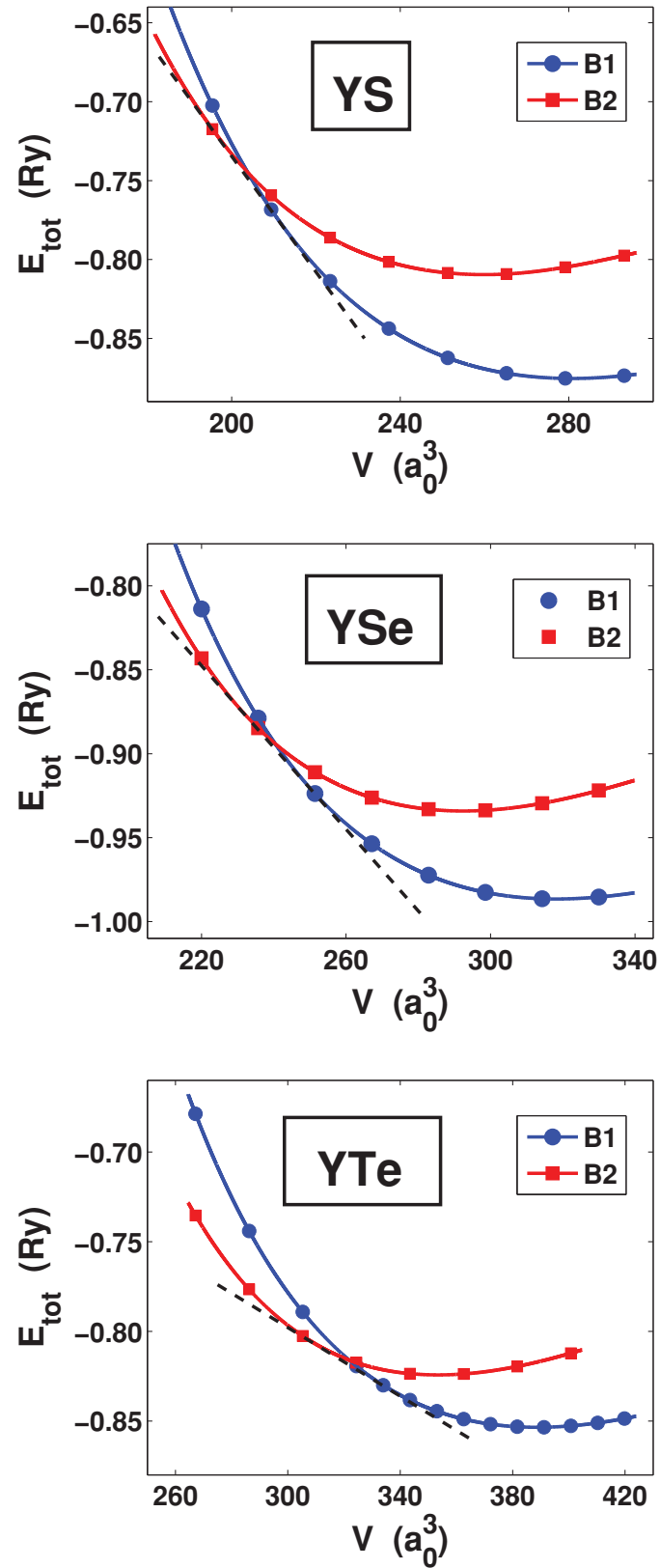

FIG. 4. (Color online) Calculated total energy as a function of volume for $\mathrm{YS}, \mathrm{YSe}$, and $\mathrm{YTe}$ in the $\mathrm{NaCl}$ and $\mathrm{CsCl}$ crystal structures.

$26 \mathrm{mRy}$ for YS, YSe, and YTe, respectively. The $\mathrm{CsCl}$ structure becomes favorable at compressed volumes, and by the construction of the common tangents of the B1 and B2 total energy curves, structural transition pressures and volume changes (pertinent to zero temperature) may be deduced. The results are summarized in Table III. The predicted transition pressures are 53, 36, and $14 \mathrm{GPa}$ for YS, YSe, and YTe, respectively.

The volume collapses associated with the transition are $7.5 \%, 7.7 \%$, and $8.1 \%$ for YS, YSe, and YTe, respectively. Since we did not consider other possible high-pressure crystal structure candidates for the yttrium monochalcogenides, we cannot exclude that a structure different from the $\mathrm{CsCl}$ structure in fact will be more favorable at high pressure.

The predicted $\mathrm{B} 1 \rightarrow \mathrm{B} 2$ transition pressures generally follow the trend observed in other alkaline earth 
TABLE III. Calculated transition pressure $P_{t}$ in GPa and volume change (in percent of the $\mathrm{B} 1$ volume at the transition pressure) for the $\mathrm{B} 1 \rightarrow \mathrm{B} 2$ structural phase transition of yttrium monochalcogenides within the GGA.

\begin{tabular}{lcc}
\hline \hline Compound & $P_{t}$ & Volume change \\
\hline YS & 53 & $7.5 \%$ \\
YSe & 36 & $7.7 \%$ \\
YTe & 14 & $8.1 \%$ \\
\hline \hline
\end{tabular}

monochalcogenides, notably lower pressures for the heavier ligands. Comparing the transition pressures of yttrium monochalcogenides with those of the neighboring alkaline earth $\mathrm{Sr}$ monochalcogenides ${ }^{10-12}$ and the isoelectronic $\mathrm{La}$ monochalcogenides, ${ }^{1}$ one observes that in the $\mathrm{Sr}$ and $\mathrm{La}$ compounds, the transition pressures are lower than those of their respective yttrium homologues, whereas the volume changes across the transition are found to be around $10 \%$, i.e., higher than those of the $\mathrm{YX}$ compounds.

Generally, for ionic compounds the observed volume changes across the $\mathrm{B} 1 \rightarrow \mathrm{B} 2$ transition are found in the range from $10 \%$ to $17 \%$ (Refs. 43-45). High-pressure measurements on monochalcogenides ${ }^{46-50}$ have revealed that in these more covalently bonded compounds, the $\mathrm{B} 1 \rightarrow \mathrm{B} 2$ transition occurs with a volume change smaller than $5 \%$. In the present work, the volume changes for the yttrium monochalcogenides are $7.5 \%-8.1 \%$, indicating that these compounds deviate slightly from being ideally ionic, but on the other hand do not fall in the range of the covalently bonded compounds either. Recently, $\mathrm{CdO}$ was also found to undergo a $\mathrm{B} 1 \rightarrow \mathrm{B} 2$ transition with a volume change across the transition of $7.3 \% .^{51}$

The transition pressure necessary to stabilize the $\mathrm{B} 2$ phase in ionic binary compounds exhibits a distinct dependence on cation and anion radius. A ratio of these radii of $r_{c} / r_{a}>0.5$ (Ref. 52) correlates with the observation of a direct B1 $\rightarrow$ B2 transition. ${ }^{53,54}$ The ratio of ionic radii $r_{c} / r_{a}$ is exactly 0.5 for YS and YSe but only 0.42 for YTe. Hence YS and YSe according to the criterion may be expected to undergo a direct $\mathrm{B} 1 \rightarrow \mathrm{B} 2$ transition, while YTe fails the criterion. However, analyzing the ratio of ionic radii for other alkaline and rareearth tellurides, we find for the sequence $\mathrm{CaTe}, \mathrm{SrTe}, \mathrm{BaTe}$, $\mathrm{LaTe}, \mathrm{CeTe}$, and PrTe values of the ratio of $0.45,0.52,0.61$, $0.47,0.46$, and 0.45 , respectively. Thus, in accordance with the rule, CaTe with $r_{c} / r_{a}<0.5$ is observed not to undergo a direct $\mathrm{B} 1 \rightarrow \mathrm{B} 2$ transition, but rather transforms to $\mathrm{B} 2$ around $35 \mathrm{GPa}$ with the occurrence of some unidentified intermediate phase around $32 \mathrm{GPa}^{12}$ On the other hand, the rare-earth tellurides LaTe, CeTe, and PrTe, for which $r_{c} / r_{a}<0.5$ as well, experimentally are found to transform directly, B1 $\rightarrow$ B2, without any intermediate phase. ${ }^{17,55-58}$ Hence, we must conclude that the rule of ratio of the ionic radii is not directly applicable to the monochalcogenides of mixed ionic-covalent character, and further experimental clarifications are needed.

The phase transformation from the $\mathrm{NaCl}$ structure to the $\mathrm{CsCl}$ structure is a typical reconstructive process, and occurs at high pressures for a large number of systems. Several mechanisms have been proposed to explain the transition. For example, in the case of $\mathrm{CaO}$, a metastable $\mathrm{Cmcm}$ phase similar to that of thallium iodide with a coordination number
7 is found to have minimum enthalpy in between the B1 to B2 high-pressure transition. ${ }^{59}$ Similar models were suggested to explain the high-pressure path of transformation for $\mathrm{NaCl}$ and $\mathrm{PbS} .{ }^{60,61}$ In the $\mathrm{YX}$ compounds, our study cannot shed light on the possible occurrence of a metastable phase lying in the energy range between the $\mathrm{NaCl}$ and $\mathrm{CsCl}$ structure and defining the path of the structural transformation from B1 to B2. Neither can we exclude a more stable high-pressure phase than B2. Further experimental investigations are necessary.

\section{BAND STRUCTURE OF YTTRIUM CHALCOGENIDES}

The calculated band structure of yttrium telluride is shown Fig. 5, without [Fig. 5(a)] and with [Fig. 5(b)] spin-orbit coupling included. The lowest-lying band around $12 \mathrm{eV}$ below the Fermi level arises from the Te $s$ states, which are separated from the Te $p$ band by a sizable gap of $8 \mathrm{eV}$. The $\mathrm{Y} d$ bands lie further above the chalcogen $p$ bands separated by a direct energy gap of the order of $2 \mathrm{eV}$. The band structures of YS and YSe are very similar. The present results are in accordance with the published band structures of YS..$^{5-8}$ The effect of spin-orbit coupling is most pronounced in the Te $p$ bands, which show
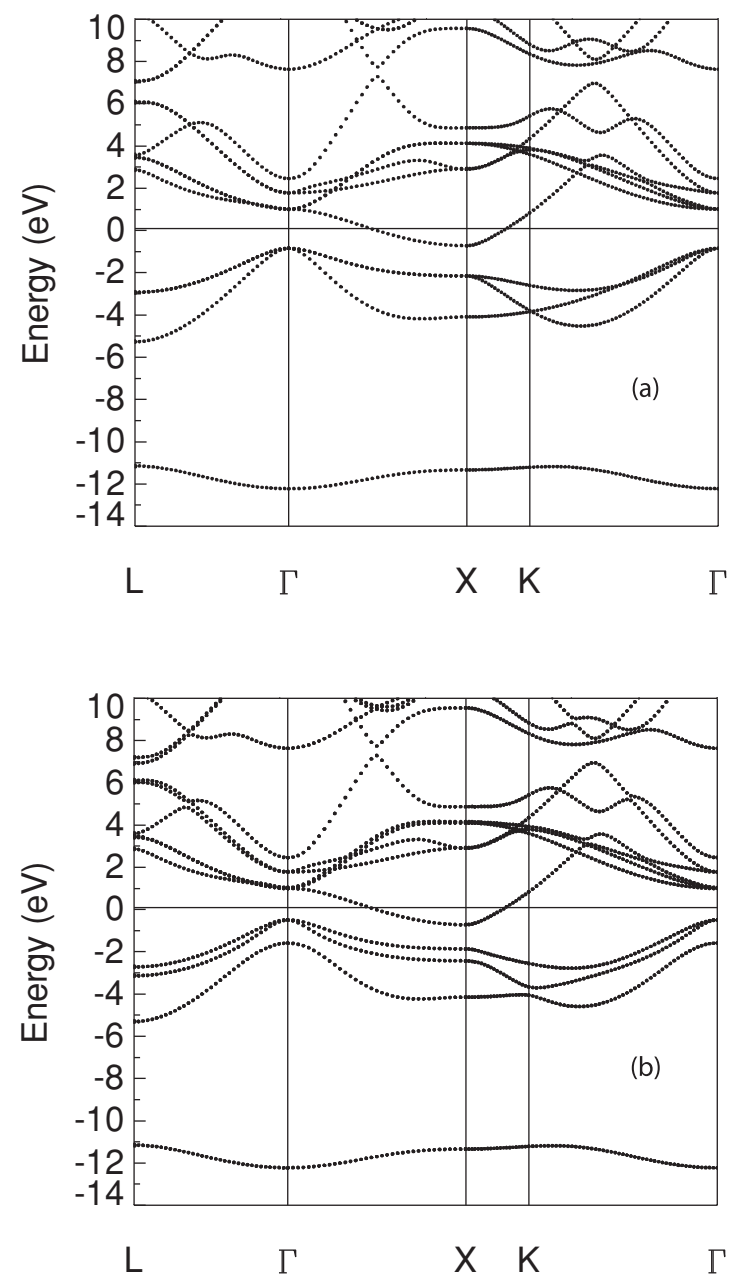

FIG. 5. Band structure of YTe, (a) without and (b) with spin-orbit interaction included. The calculations are for the $\mathrm{NaCl}$ structure at the experimental lattice constant. The Fermi level is marked with a horizontal line. 

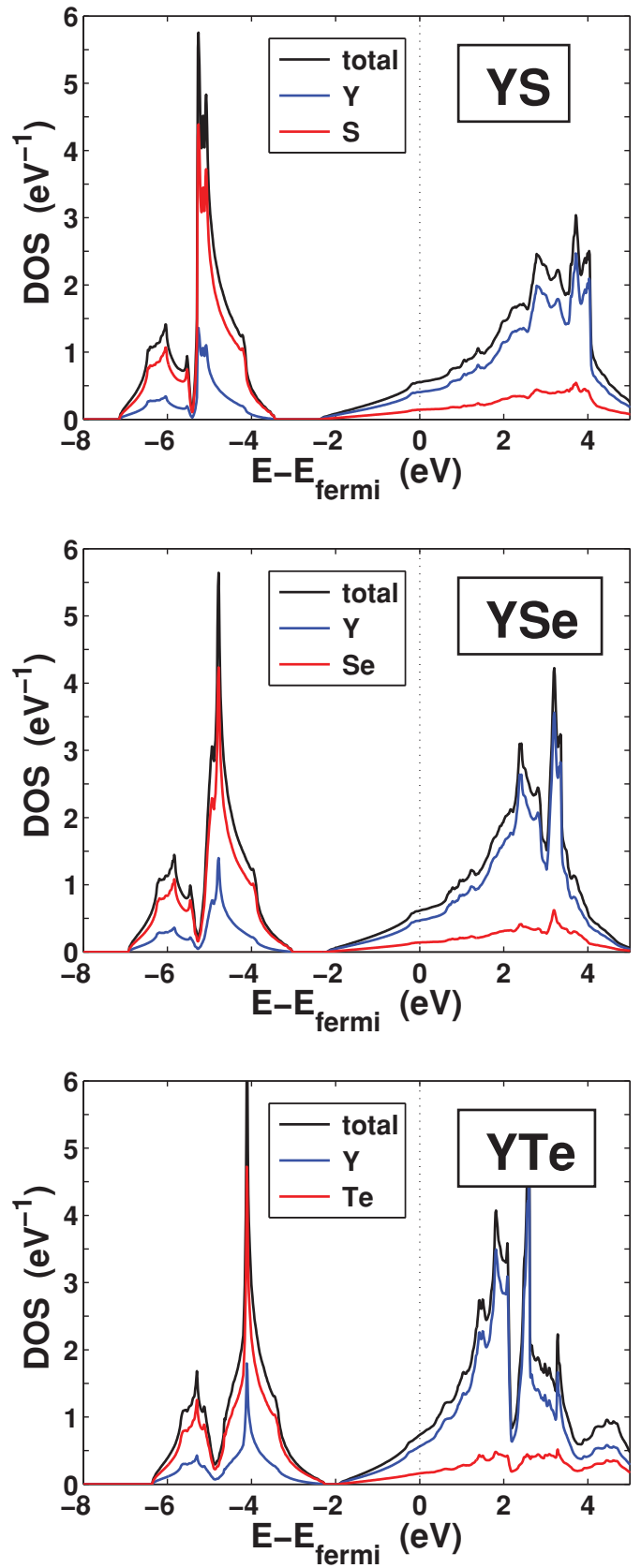

FIG. 6. (Color online) Calculated densities of states for YS, YSe, and $\mathrm{YTe}$ in the $\mathrm{NaCl}$ structure in units of states per $\mathrm{eV}$, per spin, and per unit cell. The zero of energy is placed at the Fermi level.

splittings of the order of $0.5 \mathrm{eV}$. The band structures of the yttrium monochalcogenides are very similar to those of their isostructural lanthanide compounds, except for the presence of the La $f$ states, which fall at a few $\mathrm{eV}$ above the Fermi level. ${ }^{24,62}$ In particular, also for $\operatorname{La} X(X=\mathrm{S}, \mathrm{Se}$, and $\mathrm{Te})$ the chalcogen $p$ and La $d$ bands are separated by a direct energy gap, ${ }^{24,63}$ which has recently been observed for lanthanum monochalcogenides using angle-resolved photoemission. ${ }^{64}$

The densities of states of the $\mathrm{Y}$ monochalcogenides are shown in Fig. 6. The chalcogen $p$ band is seen to shift closer to the Fermi level as we move from $\mathrm{S}$ to $\mathrm{Se}$ to $\mathrm{Te}$, and the energy gap between the chalcogen $p$ band and the $\mathrm{Y} d$ band decreases in the same sequence, in accordance with the trend

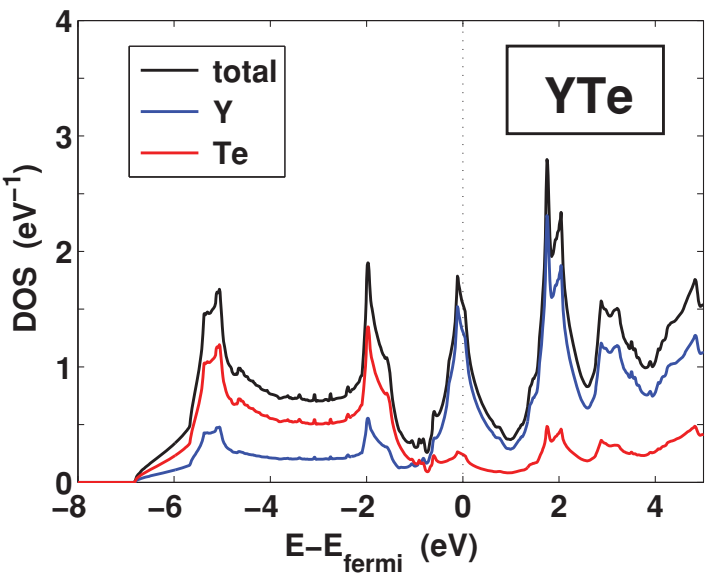

FIG. 7. (Color online) Calculated density of states for YTe in the $\mathrm{CsCl}$ structure at the volume corresponding to the transition pressure $P_{t}=14 \mathrm{GPa}$. The zero of energy is placed at the Fermi level.

observed for LaX compounds. ${ }^{64}$ The calculated densities of states at the Fermi level are found to be 12.0, 13.3, and 15.4 states/Ry per unit cell for YS, YSe, and YTe, respectively. The trend of increasing density of states at the Fermi level with heavier ligand is also found in the $\mathrm{La} X$ series, ${ }^{24,65}$ and simply reflects the decreasing width of the $\mathrm{Y} d$ bands with increasing volume. In the $\mathrm{CsCl}$ structure, the hybridization between the $\mathrm{Y} d$ and the chalcogen $p$ states is stronger, and these bands are no longer separated by an energy gap. This is illustrated for YTe in Fig. 7, which shows the density of states in the $\mathrm{CsCl}$ structure at the volume corresponding to the structural transition. A peak is seen to appear very close to the Fermi level, which might indicate that the $\mathrm{CsCl}$ structure is in fact unstable toward a symmetry-lowering distortion.

\section{CONCLUSION}

The ambient and high-pressure properties of yttrium monochalcogenides have been investigated by experiment and theory. Bulk moduli of 93, 82, and $67 \mathrm{GPa}$ are found for YS, YSe, and YTe, respectively. The compression curves have been determined for pressures up to $23 \mathrm{GPa}$ for YS and YSe, and up to $p=15 \mathrm{GPa}$ for YTe. While the experiments do not find any structural transformation in the accessible pressure range, the calculations find that a structural transition will occur under high pressure. Assuming the $\mathrm{CsCl}$ structure as an obvious candidate for the high-pressure phase, transition pressures of 53, 36, and $14 \mathrm{GPa}$ for YS, YSe, and YTe, respectively, are predicted. Since the experiment for YTe only went to a pressure of $15 \mathrm{GPa}$, it is possible that the transition has just been missed. It is thus necessary to extend the present experiments to higher pressures to test the theoretical prediction. From the band structure of the compounds in the $\mathrm{NaCl}$ structure at ambient pressure, the $\mathrm{Y} d$ and chalcogen $p$ bands are well separated, which suggests a large ionic character of the bonding in the yttrium chalcogenides. In the high-pressure $\mathrm{CsCl}$ phase, the $\mathrm{Y} d$ and chalcogen $p$ bands overlap and hybridize strongly, suggesting transformation to a more metallic bonding character. 


\section{ACKNOWLEDGMENTS}

We thank HASYLAB-DESY for permission to use the synchrotron radiation facility. J.S.O. and L.G. gratefully acknowledge financial support from the Danish Natural Sciences Research Council through DANSCATT. G.V. acknowledges CMSD University of Hyderabad for providing the computational facility.
${ }^{1}$ G. Vaitheeswaran, V. Kanchana, S. Heathman, M. Idiri, T. Le Bihan, A. Svane, A. Delin, and B. Johansson, Phys. Rev. B 75, 184108 (2007).

${ }^{2}$ S. V. Vonsovsky, Yu. A. Izyumov, and E. Z. Kumaev, Superconductivity of Transition Metals, Their Alloys and Compounds (Springer, Berlin, 1982).

${ }^{3}$ F. Hulliger and G. W. Hull Jr., Solid State Commun. 8, 1379 (1970).

${ }^{4}$ P. Roedhammer, W. Reichardt, and F. Holtzberg, Phys. Rev. Lett. 40, 465 (1978).

${ }^{5}$ M. Gupta, Phys. Rev. B 20, 4334 (1979).

${ }^{6}$ K. Schwarz and E. Wimmer, J. Phys. F 10, 1001 (1980).

${ }^{7}$ M. M. Steiner, H. Eschrig, and R. Monnier, Phys. Rev. B 45, 7183 (1992).

${ }^{8}$ H. M. Tütüncü and G. P. Srivastava, Philos. Mag. 87, 4109 (2007).

${ }^{9}$ H. Luo, R. G. Greene, K. Ghandehari, T. Li, and A. L. Ruoff, Phys. Rev. B 50, 16232 (1994).

${ }^{10}$ H. Luo, R. G. Greene, and A. L. Ruoff, Phys. Rev. B 49, 15341 (1994)

${ }^{11}$ K. Syassen, Phys. Status Solidi A 91, 11 (1985).

${ }^{12}$ H. G. Zimmer, H. Winzen, and K. Syassen, Phys. Rev. B 32, 4066 (1985).

${ }^{13}$ S. Yamaoka, O. Shimomura, H. Nakazawa, and O. Fukunaga, Solid State Commun. 33, 87 (1980).

${ }^{14}$ T. A. Grzybowski and A. L. Ruoff, Phys. Rev. B 27, 6502 (1983).

${ }^{15}$ T. A. Grzybowski and A. L. Ruoff, Phys. Rev. Lett. 53, 489 (1984).

${ }^{16}$ J. M. Léger and A. M. Redon, J. Less-Common Met. 156, 137 (1989).

${ }^{17}$ J. M. Léger, R. Epain, J. Loriers, D. Ravot, and J. Rossat-Mignod, Phys. Rev. B 28, 7125 (1983).

${ }^{18}$ J. M. Léger, Physica B 190, 84 (1993).

${ }^{19}$ T. Le Bihan, S. Darracq, S. Heathman, U. Benedict, K. Mattenberger, and O. Vogt, J. Alloys Compd. 226, 143 (1995).

${ }^{20}$ A. Jayaraman, A. K. Singh, A. Chatterjee, and S. Usha Devi, Phys. Rev. B 9, 2513 (1974).

${ }^{21}$ A. Werner, H. D. Hochheimer, and A. Jayaraman, Solid State Commun. 38, 325 (1981).

${ }^{22}$ K. Syassen, Physica B 139/140, 277 (1986).

${ }^{23}$ A. Svane, W. Temmerman, and Z. Szotek, Phys. Rev. B 59, 7888 (1999).

${ }^{24}$ G. Vaitheeswaran, V. Kanchana, and M. Rajagopalan, J. Phys. Chem. Solids 64, 15 (2003).

${ }^{25}$ G. Vaitheeswaran, L. Petit, A. Svane, V. Kanchana, and M. Rajagopalan, J. Phys. Condens. Matter 16, 4429 (2004).

${ }^{26}$ J. S. Olsen, L. Gerward, U. Benedict, H. Luo, and O. Vogt, High Temp.-High Press. 20, 553 (1988).

${ }^{27}$ H. K. Mao, J. Xu, and P. M. Bell, J. Geophys. Res. 91, 4673 (1986).

${ }^{28}$ P.-E. Werner, Arkiv för Kemi 31, 513 (1969).

${ }^{29}$ F. Birch, Phys. Rev. 54, 809 (1947).

${ }^{30}$ O. K. Andersen, Phys. Rev. B 12, 3060 (1975).

${ }^{31}$ S. Y. Savrasov, Phys. Rev. B 54, 16470 (1996).
${ }^{32}$ J. P. Perdew, K. Burke, and M. Ernzerhof, Phys. Rev. Lett. 77, 3865 (1996).

${ }^{33}$ R. A. Pollak, F. Holtzberg, J. L. Freeouf, and D. E. Eastman, Phys. Rev. Lett. 33, 820 (1974).

${ }^{34}$ R. L. Melcher, G. Güntherodt, T. Penney, and F. Holtzberg, Proceedings of the IEEE Ultrasonics Symposium, Los Angeles (IEEE, Piscataway, NJ, 1975), pp. 616; see also G. Güntherodt, R. L. Melcher, T. Penney, and F. Holtzberg, J. Magn. Magn. Mater. 3, 93 (1976).

${ }^{35}$ M. Picon and M. Patrie, C. R. Seances Acad. Sci. (Paris) 242, 1321 (1956).

${ }^{36}$ O. D. McMasters and K. A. Gschneidner Jr., AIME Nucl. Met IMD Spec. Rep. Ser. 13 10, 93 (1964).

${ }^{37}$ L. H. Brixner, J. Inorg. Nucl. Chem. 15, 199 (1960).

${ }^{38}$ S. Usha Devi and A. K. Singh, Pramana 17, 461 (1981).

${ }^{39}$ A. Jayaraman, B. Batlogg, R. G. Maines, and H. Bach, Phys. Rev. B 26, 3347 (1982).

${ }^{40}$ P. Dernier, W. Weber, and L. D. Longinotti, Phys. Rev. B 14, 3635 (1976).

${ }^{41}$ L. Gerward, J. S. Olsen, U. Benedict, S. Dabos, and O. Vogt, High Press. Res. 1, 235 (1989).

${ }^{42}$ S. von Molnar, T. Penney, and F. Holtzberg, J. Phys. (Paris) Colloq. 37, C4-241 (1976).

${ }^{43}$ C. E. Weir and G. J. Piermarini, J. Res. Natl. Bur. Stand., Sect. A 68, 105 (1964).

${ }^{44}$ S. N. Vaidya and G. C. Kennedy, J. Phys. Chem. Solids 32, 951 (1971).

${ }^{45}$ G. J. Piermarini and C. E. Weir, J. Chem. Phys. 37, 1887 (1962).

${ }^{46}$ H. Wiedemeier and H. G. von Schnering, Z. Kristallogr. 148, 295 (1978).

${ }^{47}$ H. G. von Schnering and H. Wiedemeier, Z. Kristallogr. 156, 143 (1981).

${ }^{48}$ T. Chattopadhyay, H. G. von Schnering, W. A. Grosshans, and W. B. Holazapfel, Physica B 139, 356 (1986).

${ }^{49}$ K. Knorr. L. Ehm, M. Hytha, B. Winkler, and W. Depmeier, Eur. Phys. J. B 31, 297 (2003).

${ }^{50}$ M. Croft and A. Jayaraman, Solid State Commun. 35, 203 (1980).

${ }^{51}$ H. Liu, H. K. Mao, M. Somayazulu, Y. Ding, Y. Meng, and D. Häusermann, Phys. Rev. B 70, 094114 (2004).

${ }^{52}$ R. D. Shannon and C. T. Prewitt, Acta. Crystallogr. B 25, 925 (1969).

${ }^{53}$ H. H. Demarest Jr., C. R. Cassell, and J. C. Jamieson, J. Phys. Chem. Solids 39, 1211 (1978).

${ }^{54}$ T. Yagi, T. Suzuki, and S. Akimoto, J. Phys. Chem. Solids 44, 135 (1983).

${ }^{55}$ A. Jayaraman, Bull. Am. Phys. Soc. 24, 397 (1978).

${ }^{56}$ A. K. Singh, A. Jayaraman, and A. Chatterjee, Solid State Commun. 9, 1459 (1971).

${ }^{57}$ A. Chatterjee, A. K. Singh, and A. Jayaraman, Phys. Rev. B 6, 2285 (1972). 
${ }^{58}$ I. Vedel, K. Oki, A. M. Redon, and J. M. Leger, Physica B 139, 361 (1986).

${ }^{59}$ M. Catti, Phys. Rev. B 68, 100101 (2003).

${ }^{60}$ H. T. Stokes, D. M. Hatch, J. Dong, and J. P. Lewis, Phys. Rev. B 69, 174111 (2004).

${ }^{61}$ P. Tolédano, K. Knorr, L. Ehm, and W. Depmeier, Phys. Rev. B 67, 144106 (2003).
${ }^{62}$ S. Sankaralingam, Ph.D. thesis, Anna University (1992).

${ }^{63}$ V. N. Antonov, B. N. Harmon, and A. N. Yaresko, Phys. Rev. B 69, 094404 (2004).

${ }^{64}$ M. Nakayama, H. Aoki, A. Ochiai, T. Ito, H. Kumigashira, T. Takahashi, and H. Harima, Phys. Rev. B 69, 155116 (2004).

${ }^{65}$ S. Sankaralingam, S. Mathi Jaya, G. Pari, and R. Asokamani, Phys. Status Solidi B 174, 435 (1992). 